\title{
OVERLAPPING OPTIMIZED SCHWARZ METHODS FOR PARABOLIC EQUATIONS IN $n$ DIMENSIONS
}

\author{
MINH-BINH TRAN \\ (Communicated by Matthew J. Gursky)
}

\begin{abstract}
We introduce in this paper a new tool to prove the convergence of the overlapping optimized Schwarz methods with multisubdomains. The technique is based on some estimates of the errors on the boundaries of the overlapping strips. Our guiding example is an $n$-dimensional linear parabolic equation.
\end{abstract}

\section{INTRODUCTION}

In pioneering work 11, 12, 13, P. L. Lions laid the foundations for the continuous approach of Schwarz algorithms. With the development of parallel computers, the interest in Schwarz methods has grown rapidly, as these methods lead to inherently parallel algorithms. However, with classical Schwarz methods, high frequency components converge very fast, while low frequency components converge slowly and that slows down the performance of the methods. By replacing Dirichlet transmission conditions in classical Schwarz methods by Robin or higher order transmission conditions, we can correct this weakness of the Schwarz method. The new methods are called optimized Schwarz methods and have been introduced in [5, 6], 10. Since then, the convergence properties of the optimized Schwarz methods have been studied thoroughly, based on the following two main tools: energy estimates and Laplace and Fourier transforms. Energy estimates allow us to study the convergence of the methods in the nonoverlapping case. With energy estimates, both linear and nonlinear problems have been studied, and optimized Schwarz methods have been proven to converge while applying to these equations (see, for example, the papers [1], 8], [9]). On the other hand, Laplace and Fourier transforms allow us to study the convergence of the overlapping optimized Schwarz methods, but for only a few simple equations, where all coefficients are constants and the second order operators are usually Laplace ones (see, for example, 2, [4], [5], [6]). The convergence problem of the overlapping domain decomposition methods with Robin transmission conditions still remains an open problem up to now.

In this paper, we introduce a new tool to prove the convergence of the optimized Schwarz methods for multisubdomains and apply it to an $n$-dimensional linear

Received by the editors September 25, 2010 and, in revised form, June 6, 2011 and September 1, 2011.

2000 Mathematics Subject Classification. Primary 35K10, 65M12.

Key words and phrases. Partial differential equations, domain decomposition methods.

The author has been partially supported by the ERC Advanced Grant FP7-246775 NUMERIWAVES. 
parabolic equation. The idea of the technique is to estimate carefully the difference between the values of the errors at the boundaries of the overlapping strips. The technique has the potential to be applied to many other kinds of partial differential equations including nonlinear ones, with classical solutions of the equation. The variational setting will be considered in a forthcoming paper (see [14). Our longterm goal is to construct some new tools to study the convergence problem of Schwarz methods, and this technique takes us a step closer to it.

\section{Problem Description AND MAin RESUlts}

We consider the following parabolic equation:

(2.1)

$$
\left\{\begin{array}{l}
\frac{\partial u}{\partial t}-\sum_{i, j=1}^{n} a_{i, j}(t) \frac{\partial^{2} u}{\partial x_{i} \partial x_{j}}+\sum_{i=1}^{n} b_{i}(t) \frac{\partial u}{\partial x_{i}}+c(t) u=f(t, x), \text { in }(0, T) \times \Omega, \\
u(x, t)=g(x, t), \text { on } \partial \Omega \times(0, T), \\
u(x, 0)=g(x, 0), \text { on } \Omega,
\end{array}\right.
$$

where $\Omega=D \times(\alpha, \beta)$ and $D$ is a bounded and smooth domain in $\mathbb{R}^{n-1}$. We impose the following conditions on the coefficients of (2.1).

(A1) For all $i, j$ in $\{1, \ldots, n\}, a_{i, j}(t)=a_{j, i}(t)$. There exists $\nu_{0}>0$ such that $A(t)=\left(a_{i, j}(t)\right) \geq \nu_{0} I$ for all $t$ in $(0, T)$ in the sense of symmetric positive definite matrices.

(A2) The functions $a_{i, j}, b_{i}, c$ are bounded in $C^{\infty}(\mathbb{R}) ; f$ and $g$ are bounded functions in $C^{\infty}(\overline{\Omega \times(0, T)})$.

With the conditions (A1) and (A2), equation (2.1) has a unique bounded solution $u$ in $C^{\infty}((0, T) \times \Omega)$. The proof of this result can be inferred from Theorems 9 and 10 , page 71 , in [3].

We now divide the domain $\Omega$ into $M$ subdomains, with $\Omega_{i}=D \times\left(a_{i}, b_{i}\right)$ and $\alpha=a_{1}<a_{2}<b_{1}<\cdots<a_{M}<b_{M-1}<b_{M}=\beta$. The optimized Schwarz algorithm solves $M$ equations in $M$ subdomains instead of solving directly the main problem (2.1). The iterate $\# k$ in the $l$-th domain, denoted by $u_{l}^{k}$, is defined by

$$
\begin{cases}\frac{\partial u_{l}^{k}}{\partial t}-\sum_{i, j=1}^{n} a_{i, j}(t) \frac{\partial^{2} u_{l}^{k}}{\partial x_{i} \partial x_{j}}+\sum_{i=1}^{n} b_{i}(t) \frac{\partial u_{l}^{k}}{\partial x_{i}}+c(t) u_{l}^{k}=f(t, x) & \text { in } \Omega_{l} \times(0, T), \\ \frac{\partial u_{l}^{k}\left(\cdot, a_{l}, \cdot\right)}{\partial x_{n}}+p u_{l}^{k}\left(\cdot, a_{l}, \cdot\right)=\frac{\partial u_{l-1}^{k-1}\left(\cdot, a_{l}, \cdot\right)}{\partial x_{n}}+p u_{l-1}^{k-1}\left(\cdot, a_{l}, \cdot\right) & \text { in } D \times(0, T), \\ \frac{\partial u_{l}^{k}\left(\cdot, b_{l}, \cdot\right)}{\partial x_{n}}+p u_{l}^{k}\left(\cdot, b_{l}, \cdot\right)=\frac{\partial u_{l+1}^{k-1}\left(\cdot, b_{l}, \cdot\right)}{\partial x_{n}}+p u_{l+1}^{k-1}\left(\cdot, b_{l}, \cdot\right) & \text { in } D \times(0, T) ;\end{cases}
$$

here, $p$ is a constant and for each vector $x$ in $\mathbb{R}^{n}$, we denote $x=\left(X, x_{n}\right)$, with $X \in \mathbb{R}^{n-1}$ and $x_{n} \in \mathbb{R}$. Each iterate inherits the boundary conditions and the initial values of $u$ :

$$
u_{l}^{k}(x, t)=g(x, t) \text { on }\left(\partial \Omega_{j} \cap \partial \Omega\right) \times(0, T), \quad u_{l}^{k}(x, 0)=g(x, 0) \text { in } \Omega_{j},
$$

and a special treatment for the extreme subdomains,

$$
u_{1}^{k}(\cdot, \alpha, \cdot)=g(\cdot, \alpha, \cdot), \quad u_{M}^{k}(\cdot, \beta, \cdot)=g(\cdot, \beta, \cdot) .
$$


A bounded initial guess $h^{0}$ in $C^{\infty}(\overline{\Omega \times(0, T)})$ is provided; i.e., we solve at the first iteration equations (2.2), with boundary data on left and right:

$$
\begin{gathered}
\frac{\partial u_{l}^{1}\left(\cdot, a_{l}, \cdot\right)}{\partial x_{n}}+p u_{l}^{1}\left(\cdot, a_{l}, \cdot\right)=h^{0}\left(\cdot, a_{l}, \cdot\right) \text { in } D \times(0, T), \\
\frac{\partial u_{l}^{1}\left(\cdot, b_{l}, \cdot\right)}{\partial x_{n}}+p u_{l}^{1}\left(\cdot, b_{l}, \cdot\right)=h^{0}\left(\cdot, b_{l}, \cdot\right) \text { in } D \times(0, T) .
\end{gathered}
$$

By using an induction argument and the same arguments as in Theorem 2, page 144, in [3], we can see that each subproblem in (2.2) in each iteration has a unique solution. Theorem 10, page 71 , in [3] shows that these solutions belong to $C^{\infty}(\Omega \times$ $(0, T))$. This means that the algorithm is well-posed.

Denote by $e_{l}^{k}$ the difference between $u_{l}^{k}$ and $u$ and subtract equation (2.2) from the main equation (2.1). We get the following equation on $e_{l}^{k}$ :

$$
\begin{cases}\frac{\partial e_{l}^{k}}{\partial t}-\sum_{i, j=1}^{n} a_{i, j}(t) \frac{\partial^{2} e_{l}^{k}}{\partial x_{i} \partial x_{j}}+\sum_{i=1}^{n} b_{i}(t) \frac{\partial e_{l}^{k}}{\partial x_{i}}+c(t) e_{l}^{k}=0 & \text { in } \Omega_{l} \times(0, T), \\ \frac{\partial e_{l}^{k}\left(\cdot, a_{l}, \cdot\right)}{\partial x_{n}}+p e_{l}^{k}\left(\cdot, a_{l}, \cdot\right)=\frac{\partial e_{l-1}^{k-1}\left(\cdot, a_{l}, \cdot\right)}{\partial x_{n}}+p e_{l-1}^{k-1}\left(\cdot, a_{l}, \cdot\right) & \text { in } D \times(0, T), \\ \frac{\partial e_{l}^{k}\left(\cdot, b_{l}, \cdot\right)}{\partial x_{n}}+p e_{l}^{k}\left(\cdot, b_{l}, \cdot\right)=\frac{\partial e_{l+1}^{k-1}\left(\cdot, b_{l}, \cdot\right)}{\partial x_{n}}+p e_{l+1}^{k-1}\left(\cdot, b_{l}, \cdot\right) & \text { in } D \times(0, T) .\end{cases}
$$

Similarly, each iterate inherits the boundary conditions and the initial values of $u$,

$$
e_{l}^{k}=0 \text { on }\left(\partial \Omega_{l} \cap \partial \Omega\right) \times(0, T), \quad e_{l}^{k}(\cdot, \cdot, 0)=0 \text { in } \Omega_{l},
$$

and the special treatment for the extreme subdomains,

$$
e_{1}^{k}(\cdot, \alpha, \cdot)=0, \quad e_{M}^{k}(\cdot, \beta, \cdot)=0 .
$$

The following theorem states that the algorithm converges.

Theorem 2.1. Letting $\varphi$ be a strictly positive function in $C^{1}(\mathbb{R})$ such that $-\max _{x_{n} \in \mathbb{R}}\left(\frac{\varphi^{\prime}}{\varphi}\left(x_{n}\right)\right)$ is large enough, the optimized Schwarz method converges in the following sense:

$$
\lim _{k \rightarrow \infty} \max _{l \in\{1, \ldots, M\}}\left\|\left(\frac{\partial\left(u_{l}^{k}-u\right)}{\partial x_{n}} \exp \left(p x_{n}\right)\right)^{2} \varphi(t)\right\|_{C\left(\overline{\Omega_{l} \times(0, T)}\right)}=0 .
$$

Moreover, for $l$ in $\{1, \ldots, M\}$, the sequence $\left\{u_{l}^{k}\right\}$ converges pointwisely to $u$ as $k$ tends to infinity.

Remark 2.2. We can see that if we choose $\varphi\left(x_{n}\right)=\exp \left(-\gamma x_{n}\right)$, then if $\gamma$ is large enough, $-\max _{x_{n} \in \mathbb{R}}\left(\frac{\varphi^{\prime}}{\varphi}\left(x_{n}\right)\right)$ is large enough. The condition of our theorem is then satisfied.

Remark 2.3. Since $a_{i, j}, b_{i}$ are functions of $t$, and the domain is divided into $n$ subdomains, we cannot use Fourier and Laplace transforms. Moreover, since the subdomains are overlapping, the energy estimate method cannot be used in our case. In the next section, we introduce a new technique to prove the convergence of the algorithm. The technique is based on the observation that we can estimate the difference between the values of $e_{l}^{k}$ on the boundary and in the interior. 
Remark 2.4. The result in the theorem remains true if we let $a_{i, j}, b_{i}$ be bounded and continuous functions of $t$ and $x$ but not depend on the $n$-th space variable $x_{n}$, as we can see in the proof in the following section.

Remark 2.5. The idea of the proof is to transform the equations in subdomains with Robin boundary conditions into equations with Dirichlet boundary conditions and then to apply the maximum principle to get some boundary estimates for the errors. However, the algorithm with Robin transmission conditions is not equivalent to the classical algorithm with Dirichlet transmission conditions, since the transformed equation is an equation on $\frac{\partial \epsilon_{l}^{k}}{\partial x_{n}}$ and the form of the operator of the equation is completely changed.

\section{The CONVERGENCE OF THE ALGORITHM}

This section is devoted to the proof of Theorem 2.1. We divide the proof into two steps.

Step 1. The error estimates.

For $k$ in $\mathbb{N}$ and $i$ in $\{1, \ldots, M\}$, setting $\epsilon_{l}^{k}$ to be $e_{l}^{k} \exp \left(p x_{n}\right)$, we get $e_{l}^{k}=$ $\epsilon_{l}^{k} \exp \left(-p x_{n}\right)$. Equation (2.3) then leads to

$$
\left\{\begin{array}{l}
\frac{\partial \epsilon_{l}^{k}}{\partial t}-\sum_{i, j=1}^{n} a_{i, j} \frac{\partial^{2} \epsilon_{l}^{k}}{\partial x_{i} \partial x_{j}}+\sum_{i=1}^{n-1}\left(p a_{i, n}+b_{i}\right) \frac{\partial \epsilon_{l}^{k}}{\partial x_{i}}+\left(2 p a_{n, n}+b_{n}\right) \frac{\partial \epsilon_{l}^{k}}{\partial x_{n}} \\
\quad+\left(c-p b_{n}-p^{2} a_{n, n}\right) \epsilon_{l}^{k}=0, \text { in } \Omega_{l} \times(0, T), \\
\frac{\partial \epsilon_{l}^{k}\left(\cdot, a_{l}, \cdot\right)}{\partial x_{n}}=\frac{\partial \epsilon_{l-1}^{k-1}\left(\cdot, a_{l}, \cdot\right)}{\partial x_{n}} \text { in } D \times(0, T), \\
\frac{\partial \epsilon_{l}^{k}\left(\cdot, b_{l}, \cdot\right)}{\partial x_{n}}=\frac{\partial \epsilon_{l+1}^{k-1}\left(\cdot, b_{l}, \cdot\right)}{\partial x_{n}} \text { in } D \times(0, T), \\
\epsilon_{l}^{k}(\cdot, \cdot, \cdot)=0 \text { on }\left(\partial \Omega_{j} \cap \partial \Omega\right) \times(0, T), \\
\epsilon_{l}^{k}(\cdot, \cdot, 0)=0 \text { in } \Omega_{j},
\end{array}\right.
$$

and for the extreme subdomains,

$$
\epsilon_{1}^{k}\left(\cdot, a_{1}, \cdot\right)=0, \quad \epsilon_{M}^{k}\left(\cdot, b_{M}, \cdot\right)=0 .
$$

Setting $\nu_{l}^{k}=\frac{\partial \epsilon_{l}^{k}}{\partial x_{n}}$, we infer from equation (3.1) that for $l$ in $\{2, \ldots, M-1\}$,

$$
\left\{\begin{array}{l}
\frac{\partial \nu_{l}^{k}}{\partial t}-\sum_{i, j=1}^{n} a_{i, j} \frac{\partial^{2} \nu_{l}^{k}}{\partial x_{i} \partial x_{j}}+\sum_{i=1}^{n-1}\left(p a_{i, n}+b_{i}\right) \frac{\partial \nu_{l}^{k}}{\partial x_{i}}+\left(2 p a_{n, n}+b_{n}\right) \frac{\partial \nu_{l}^{k}}{\partial x_{n}} \\
\quad+\left(c-p b_{n}-p^{2} a_{n, n}\right) \nu_{l}^{k}=0, \text { in } \Omega_{l} \times(0, T), \\
\nu_{l}^{k}\left(\cdot, a_{l}, \cdot\right)=\nu_{l-1}^{k-1}\left(\cdot, a_{l}, \cdot\right) \text { in } D \times(0, T), \\
\nu_{l}^{k}\left(\cdot, b_{l}, \cdot\right)=\nu_{l+1}^{k-1}\left(\cdot, b_{l}, \cdot\right) \text { in } D \times(0, T), \\
\nu_{l}^{k}(\cdot, \cdot, \cdot)=0 \text { on }\left(\partial \Omega_{j} \cap \partial \Omega\right) \times(0, T), \\
\nu_{l}^{k}(\cdot, \cdot, 0)=0 \text { in } \Omega_{j} .
\end{array}\right.
$$

On $\overline{\Omega_{l} \times(0, T)}$, we define $\Phi=\left(\nu_{l}^{k}\right)^{2} \phi\left(x_{n}\right) \varphi(t)$, where $\phi$ is a strictly positive function in $C^{2}(\mathbb{R})$ to be chosen later, with the observation that $-\max _{x_{n} \in \mathbb{R}}\left(\frac{\varphi^{\prime}}{\varphi}\left(x_{n}\right)\right)$ is large enough. Our purpose is to construct an operator $\mathfrak{L}$ of $\Phi$, such that $\mathfrak{L}(\Phi)$ is 
negative and then on $\mathfrak{L}$, we can apply the maximum principle to get some estimates on the boundaries for $\Phi$. We now consider the following operator:

$$
\mathfrak{L}_{0}(\Phi):=\frac{\partial \Phi}{\partial t}-\sum_{i, j=1}^{n} a_{i, j} \frac{\partial^{2} \Phi}{\partial x_{i} \partial x_{j}} .
$$

A simple calculation gives

$$
\begin{aligned}
\mathfrak{L}_{0}(\Phi) & =2 \nu_{l}^{k} \phi \varphi\left(\frac{\partial \nu_{l}^{k}}{\partial t}-\sum_{i, j=1}^{n} a_{i, j} \frac{\partial^{2} \nu_{l}^{k}}{\partial x_{i} x_{j}}\right)-\sum_{i, j=1}^{n} 2 a_{i, j} \phi \varphi \frac{\partial \nu_{l}^{k}}{\partial x_{i}} \frac{\partial \nu_{l}^{k}}{\partial x_{j}} \\
& -\sum_{i=1}^{n} 2 a_{i, n} \phi^{\prime} \varphi \nu_{l}^{k} \frac{\partial \nu_{l}^{k}}{\partial x_{i}}+\left(\frac{\varphi^{\prime}}{\varphi}-a_{n, n} \frac{\phi^{\prime \prime}}{\phi}\right) \phi \varphi\left(\nu_{l}^{k}\right)^{2} .
\end{aligned}
$$

We observe that the second term on the right-hand side of the previous inequality is negative. It directly leads to

$$
\begin{aligned}
\mathfrak{L}_{0}(\Phi) & \leq 2 \nu_{l}^{k} \phi \varphi\left(\frac{\partial \nu_{l}^{k}}{\partial t}-\sum_{i, j=1}^{n} a_{i, j} \frac{\partial^{2} \nu_{l}^{k}}{\partial x_{i} x_{j}}\right) \\
& -\sum_{i=1}^{n} 2 a_{i, n} \phi^{\prime} \varphi \nu_{l}^{k} \frac{\partial \nu_{l}^{k}}{\partial x_{i}}+\left(\frac{\varphi^{\prime}}{\varphi}-a_{n, n} \frac{\phi^{\prime \prime}}{\phi}\right) \phi \varphi\left(\nu_{l}^{k}\right)^{2} .
\end{aligned}
$$

We now replace (3.2) into (3.5) and get the following bound for $\mathfrak{L}_{0}(\Phi)$ :

$$
\begin{aligned}
2 \nu_{l}^{k} \phi \varphi & \left(-\sum_{i=1}^{n-1}\left(p a_{i, n}+b_{i}\right) \frac{\partial \nu_{l}^{k}}{\partial x_{i}}-\left(2 p a_{n, n}+b_{n}\right) \frac{\partial \nu_{l}^{k}}{\partial x_{n}}-\left(c-p b_{n}-p^{2} a_{n, n}\right) \nu_{l}^{k}\right) \\
& -\sum_{i=1}^{n} 2 a_{i, n} \phi^{\prime} \varphi \nu_{l}^{k} \frac{\partial \nu_{l}^{k}}{\partial x_{i}}+\left(\frac{\varphi^{\prime}}{\varphi}-a_{n, n} \frac{\phi^{\prime \prime}}{\phi}\right) \phi \varphi\left(\nu_{l}^{k}\right)^{2} .
\end{aligned}
$$

Substituting

$$
\frac{\partial \Phi}{\partial x_{i}}=2 \phi \varphi \nu_{l}^{k} \frac{\partial \nu_{l}^{k}}{\partial x_{i}}, \text { for } i \in\{1, \ldots, n-1\}
$$

and

$$
\frac{\partial \Phi}{\partial x_{n}}=2 \phi \varphi \nu_{l}^{k} \frac{\partial \nu_{l}^{k}}{\partial x_{n}}+\phi^{\prime} \varphi\left(\nu_{l}^{k}\right)^{2}
$$

into (3.6), we get

$$
\begin{aligned}
& \frac{\partial \Phi}{\partial t}-\sum_{i, j=1}^{n} a_{i, j} \frac{\partial^{2} \Phi}{\partial x_{i} \partial x_{j}}+\left(2 p a_{n, n}+b_{n}+a_{n, n} \frac{\phi^{\prime}}{\phi}\right) \frac{\partial \Phi}{\partial x_{n}} \\
& +\sum_{i=1}^{n-1}\left(p a_{i, n}+b_{i}+a_{i n} \frac{\phi^{\prime}}{\phi}\right) \frac{\partial \Phi}{\partial x_{i}} \\
& \leq \phi \varphi\left(\nu_{l}^{k}\right)^{2}\left(\frac{\varphi^{\prime}}{\varphi}-a_{n n} \frac{\phi^{\prime \prime}}{\phi}+a_{n, n} \frac{\phi^{\prime 2}}{\phi^{2}}-2\left(c-p b_{n}-p^{2} a_{n, n}\right)+\left(2 p a_{n, n}+b_{n}\right) \frac{\phi^{\prime}}{\phi}\right) .
\end{aligned}
$$


We now get the formula for $\mathfrak{L}$ :

$$
\begin{aligned}
\mathfrak{L}(\Phi) & :=\frac{\partial \Phi}{\partial t}-\sum_{i, j=1}^{n} a_{i, j} \frac{\partial^{2} \Phi}{\partial x_{i} \partial x_{j}}+\left(2 p a_{n, n}+b_{n}+a_{n n} \frac{\phi^{\prime}}{\phi}\right) \frac{\partial \Phi}{\partial x_{n}} \\
& +\sum_{i=1}^{n-1}\left(p a_{i, n}+b_{i}+a_{i n} \frac{\phi^{\prime}}{\phi}\right) \frac{\partial \Phi}{\partial x_{i}} .
\end{aligned}
$$

Then if we choose $\varphi$ such that $-\max _{x_{n} \in \mathbb{R}}\left(\frac{\varphi^{\prime}}{\varphi}\left(x_{n}\right)\right)$ is large enough, since $a_{n, n}, b_{n}$, $c, \frac{\phi^{\prime}}{\phi}, \frac{\phi^{\prime \prime}}{\phi}$ are all bounded in $C(\mathbb{R})$, we can obtain a negative sign on the right-hand side of (3.7), which means that $\mathfrak{L}(\Phi)$ is negative.

Since $\mathfrak{L}(\Phi) \leq 0$, the maximum of $\Phi$ can only be attained on the boundary of $\Omega_{l} \times(0, T)$. Using the fact that $\Phi=0$ on $\partial \Omega_{l} \cap \partial \Omega$ and on $\Omega \times\{0\}$, we have the following three estimates.

Estimate 1. $1 \leq l \leq M$.

The maximum value of $\Phi$ can be achieved on both $\bar{D} \times\left\{a_{l}\right\} \times[0, T]$ and $\bar{D} \times$ $\left\{b_{l}\right\} \times[0, T]:$

$$
\begin{aligned}
& \left(\nu_{l}^{k}\left(X, x_{n}, t\right)\right)^{2} \phi\left(x_{n}\right) \varphi(t) \\
& \quad \leq \max \left\{\max _{\bar{D} \times[0, T]}\left\{\left(\nu_{l}^{k}\left(X, a_{l}, t\right)\right)^{2} \phi\left(a_{l}\right) \varphi(t)\right\}, \max _{\bar{D} \times[0, T]}\left\{\left(\nu_{l}^{k}\left(X, b_{l}, t\right)\right)^{2} \phi\left(b_{l}\right) \varphi(t)\right\}\right\} .
\end{aligned}
$$

Estimate 2. $l=1$.

The maximum value of $\Phi$ can be achieved on both $\bar{D} \times\left\{a_{1}\right\} \times[0, T]$ and $\bar{D} \times$ $\left\{b_{1}\right\} \times[0, T]$. If the maximum of $\Phi$ is achieved on $\bar{D} \times\left\{a_{1}\right\} \times[0, T]$, then at the maximum point, we need that $\frac{\partial \Phi}{\partial n}>0$ due to Hopf's Lemma. We compute

$$
\begin{aligned}
\frac{\partial \Phi}{\partial n}\left(., a_{1}, t\right) & =-\frac{\partial \nu_{1}^{k}}{\partial x_{n}} \nu_{1}^{k} \phi\left(a_{1}\right) \varphi(t)-\left(\nu_{1}^{k}\right)^{2} \phi^{\prime}\left(a_{1}\right) \varphi(t) \\
& =-\phi\left(a_{1}\right) \varphi(t)\left[\frac{\partial^{2} \epsilon_{1}^{k}}{\partial x_{n}^{2}} \frac{\partial \epsilon_{1}^{k}}{\partial x_{n}}+\left(\frac{\partial \epsilon_{1}^{k}}{\partial x_{n}}\right)^{2} \frac{\phi^{\prime}\left(a_{1}\right)}{\phi\left(a_{1}\right)}\right] .
\end{aligned}
$$

Since

$$
\begin{array}{r}
\frac{\partial \epsilon_{1}^{k}}{\partial t}\left(., a_{1}, .\right)-\sum_{i, j=1}^{n} a_{i, j} \frac{\partial^{2} \epsilon_{1}^{k}}{\partial x_{i} \partial x_{j}}\left(., a_{1}, .\right)+\sum_{i=1}^{n-1}\left(p a_{i, n}+b_{i}\right) \frac{\partial \epsilon_{1}^{k}}{\partial x_{i}}\left(., a_{1}, .\right) \\
+\left(2 p a_{n, n}+b_{n}\right) \frac{\partial \epsilon_{1}^{k}}{\partial x_{n}}\left(., a_{1}, .\right)+\left(c-p b_{n}-p^{2} a_{n, n}\right) \epsilon_{1}^{k}\left(., a_{1}, .\right)=0, \text { in } D \times(0, T),
\end{array}
$$

and $\epsilon_{l}^{k}\left(., a_{1},.\right)=0$ on $D \times(0, T)$, we deduce

$$
-\frac{\partial^{2} \epsilon_{1}^{k}}{\partial x_{n}^{2}}\left(., a_{1}, .\right)+\left(2 p+\frac{b_{n}}{a_{n, n}}\right) \frac{\partial \epsilon_{1}^{k}}{\partial x_{n}}\left(., a_{1}, .\right)=0,
$$

and as a consequence, we can write $\frac{\partial \Phi}{\partial n}$ in a different way:

$$
\frac{\partial \Phi}{\partial n}\left(., a_{1}, .\right)=-\phi\left(a_{1}\right) \varphi(t)\left(\frac{\partial \epsilon_{1}^{k}}{\partial x_{n}}\right)^{2}\left[\left(2 p+\frac{b_{n}}{a_{n, n}}\right)+\frac{\phi^{\prime}\left(a_{1}\right)}{\phi\left(a_{1}\right)}\right] .
$$


Choosing $\phi$ such that

$$
\left(2 p+\frac{b_{n}(t)}{a_{n, n}(t)}\right)+\frac{\phi^{\prime}\left(a_{1}\right)}{\phi\left(a_{1}\right)}>0
$$

we can see that

$$
\frac{\partial \Phi}{\partial n}\left(., a_{1}, .\right)<0
$$

which means that the maximum of $\Phi$ can be achieved only on $\bar{D} \times\left\{b_{1}\right\} \times[0, T]$. Then

$$
\left(\nu_{1}^{k}\left(X, x_{n}, t\right)\right)^{2} \phi\left(x_{n}\right) \varphi(t) \leq \max _{\bar{D} \times[0, T]}\left\{\left(\nu_{1}^{k}\left(X, b_{1}, t\right)\right)^{2} \phi\left(b_{1}\right) \varphi(t)\right\} .
$$

Estimate 3. $l=M$.

The maximum value(s) of $\Phi$ can be achieved on both $\bar{D} \times\left\{a_{M}\right\} \times[0, T]$ and $\bar{D} \times\left\{b_{M}\right\} \times[0, T]$. If the maximum of $\Phi$ is achieved on $\bar{D} \times\left\{b_{M}\right\} \times[0, T]$, then at the maximum point, we need that $\frac{\partial \Phi}{\partial n}>0$ due to Hopf's Lemma. Similarly as in Estimate 2, we can get

$$
\begin{aligned}
\frac{\partial \Phi}{\partial n}\left(., b_{M}, t\right) & =\frac{\partial \nu_{1}^{k}}{\partial x_{n}} \nu_{1}^{k} \phi\left(b_{M}\right) \varphi(t)+\left(\nu_{1}^{k}\right)^{2} \phi^{\prime}\left(b_{M}\right) \varphi(t) \\
& =\phi\left(b_{M}\right) \varphi(t)\left(\frac{\partial \epsilon_{1}^{k}}{\partial x_{n}}\right)^{2}\left[\left(2 p+\frac{b_{n}}{a_{n, n}}\right)+\frac{\phi^{\prime}\left(b_{M}\right)}{\phi\left(b_{M}\right)}\right] .
\end{aligned}
$$

With the function $\phi$ satisfying

$$
\left(2 p+\frac{b_{n}(t)}{a_{n, n}(t)}\right)+\frac{\phi^{\prime}\left(b_{M}\right)}{\phi\left(b_{M}\right)}<0
$$

we can see that

$$
\frac{\partial \Phi}{\partial n}\left(., b_{M}, .\right)<0
$$

which means that the maximum of $\Phi$ can be achieved only on $\bar{D} \times\left\{a_{M}\right\} \times[0, T]$. Then

$$
\left(\nu_{M}^{k}\left(X, x_{n}, t\right)\right)^{2} \phi\left(x_{n}\right) \varphi(t) \leq \max _{\bar{D} \times[0, T]}\left\{\left(\nu_{M}^{k}\left(X, a_{M}, t\right)\right)^{2} \phi\left(a_{M}\right) \varphi(t)\right\}
$$

Step 2. Proof of convergence,

$$
\lim _{k \rightarrow \infty} \max _{l \in\{1, \ldots, M\}}\left\|\left(\nu_{l}^{k}\right)^{2} \varphi(t)\right\|_{C\left(\overline{\Omega_{l} \times(0, T)}\right)}=0 .
$$

In the proof of convergence, we will use the three estimates (3.9), (3.10) and (3.11) by fixing $\varphi$ and replacing $\phi$ by appropriate functions $\bar{\phi}_{i}, \tilde{\phi}_{i}, \bar{\phi}_{*}, \tilde{\phi}_{*}(i \in$ $\{1, \ldots, M\})$ in each subdomain. We define

$$
E_{k}=\max _{l \in\{1, \ldots, M\}}\left\|\left(\nu_{l}^{k}\right)^{2} \phi \varphi\right\|_{C\left(\overline{\Omega_{l} \times(0, T)}\right)} .
$$

Step 2.1. Estimate of the right boundaries of the subdomains.

Consider the $M$-th domain. At the $k$-th step, (3.11) implies

$$
\left(\nu_{M}^{k}\left(X, x_{n}, t\right)\right)^{2} \bar{\phi}_{M}\left(x_{n}\right) \varphi(t) \leq \max _{\bar{D} \times[0, T]}\left\{\left(\nu_{M}^{k}\left(X, a_{M}, t\right)\right)^{2} \bar{\phi}_{M}\left(a_{M}\right) \varphi(t)\right\},
$$

where $\bar{\phi}_{M}$ is a strictly positive function and will be chosen later. 
Replacing $x_{n}$ by $b_{M-1}$, we get

$$
\left(\nu_{M}^{k}\left(X, b_{M-1}, t\right)\right)^{2} \bar{\phi}_{M}\left(b_{M-1}\right) \varphi(t) \leq \max _{\bar{D} \times[0, T]}\left\{\left(\nu_{M}^{k}\left(X, a_{M}, t\right)\right)^{2} \bar{\phi}_{M}\left(a_{M}\right) \varphi(t)\right\} .
$$

Since $\nu_{M}^{k}\left(X, b_{M-1}, t\right)=\nu_{M-1}^{k+1}\left(X, b_{M-1}, t\right)$,

$$
\left(\nu_{M-1}^{k+1}\left(X, b_{M-1}, t\right)\right)^{2} \bar{\phi}_{M}\left(b_{M-1}\right) \varphi(t) \leq \max _{\bar{D} \times[0, T]}\left\{\left(\nu_{M}^{k}\left(X, a_{M}, t\right)\right)^{2} \bar{\phi}_{M}\left(a_{M}\right) \varphi(t)\right\} .
$$

The inequality becomes

$$
\left(\nu_{M-1}^{k+1}\left(X, b_{M-1}, t\right)\right)^{2} \varphi(t) \leq \frac{\bar{\phi}_{M}\left(a_{M}\right)}{\bar{\phi}_{M}\left(b_{M-1}\right)} \max _{\bar{D} \times[0, T]}\left\{\left(\nu_{M}^{k}\left(X, a_{M}, t\right)\right)^{2} \varphi(t)\right\} .
$$

We can choose $\bar{\phi}_{M}$ such that $\frac{\bar{\phi}_{M}\left(a_{M}\right)}{\bar{\phi}_{M}\left(b_{M-1}\right)}<1$ and deduce

$$
\left(\nu_{M-1}^{k+1}\left(X, b_{M-1}, t\right)\right)^{2} \varphi(t) \leq \frac{\bar{\phi}_{M}\left(a_{M}\right)}{\bar{\phi}_{M}\left(b_{M-1}\right)} E_{k} .
$$

Moreover, on the $(M-1)$-th domain, at the $(k+1)$-th step, (3.9) leads to

$$
\begin{aligned}
& \left(\nu_{M-1}^{k+1}\left(X, x_{n}, t\right)\right)^{2} \bar{\phi}_{M-1}\left(x_{n}\right) \varphi(t) \\
& \leq \max \left\{\max _{\bar{D} \times[0, T]}\left\{\left(\nu_{M-1}^{k+1}\left(X, b_{M-1}, t\right)\right)^{2} \bar{\phi}_{M-1}\left(b_{M-1}\right) \varphi(t)\right\},\right. \\
& \left.\max _{\bar{D} \times[0, T]}\left\{\left(\nu_{M-1}^{k+1}\left(X, a_{M-1}, t\right)\right)^{2} \bar{\phi}_{M-1}\left(a_{M-1}\right) \varphi(t)\right\}\right\},
\end{aligned}
$$

where $\bar{\phi}_{M-1}$ is a strictly positive function that will be chosen later.

Since $\nu_{M-1}^{k+1}\left(X, b_{M-2}, t\right)=\nu_{M-2}^{k+2}\left(X, b_{M-2}, t\right)$,

$$
\begin{aligned}
& \left(\nu_{M-2}^{k+2}\left(X, b_{M-2}, t\right)\right)^{2} \bar{\phi}_{M-1}\left(b_{M-2}\right) \varphi(t) \\
& \leq \max \left\{\max _{\bar{D} \times[0, T]}\left\{\left(\nu_{M-1}^{k+1}\left(X, b_{M-1}, t\right)\right)^{2} \bar{\phi}_{M-1}\left(b_{M-1}\right) \varphi(t)\right\},\right. \\
& \left.\max _{\bar{D} \times[0, T]}\left\{\left(\nu_{M-1}^{k+1}\left(X, a_{M-1}, t\right)\right)^{2} \bar{\phi}_{M-1}\left(a_{M-1}\right) \varphi(t)\right\}\right\} .
\end{aligned}
$$

Hence

$$
\begin{aligned}
& \left(\nu_{M-2}^{k+2}\left(X, b_{M-2}, t\right)\right)^{2} \varphi(t) \\
& \leq \max \left\{\frac{\bar{\phi}_{M-1}\left(b_{M-1}\right)}{\bar{\phi}_{M-1}\left(b_{M-2}\right)} \max _{\bar{D} \times[0, T]}\left\{\left(\nu_{M-1}^{k+1}\left(X, b_{M-1}, t\right)\right)^{2} \varphi(t)\right\},\right. \\
& \left.\max _{\bar{D} \times[0, T]}\left(\frac{\bar{\phi}_{M-1}\left(a_{M-1}\right)}{\bar{\phi}_{M-1}\left(b_{M-2}\right)}\left(\nu_{M-1}^{k+1}\left(X, a_{M-1}, t\right)\right)^{2} \varphi(t)\right)\right\} .
\end{aligned}
$$

Combining this inequality with (3.12), we get

$$
\left(\nu_{M-2}^{k+2}\left(X, b_{M-2}, t\right)\right)^{2} \varphi(t) \leq \max \left\{\frac{\bar{\phi}_{M-1}\left(b_{M-1}\right)}{\bar{\phi}_{M-1}\left(b_{M-2}\right)} \frac{\bar{\phi}_{M}\left(a_{M}\right)}{\bar{\phi}_{M}\left(b_{M-1}\right)} E_{k}, \frac{\bar{\phi}_{M-1}\left(a_{M-1}\right)}{\bar{\phi}_{M-1}\left(b_{M-2}\right)} E_{k+1}\right\} .
$$

Choosing $\bar{\phi}_{M-1}$ such that

$$
\frac{\bar{\phi}_{M-1}\left(b_{M-1}\right)}{\bar{\phi}_{M-1}\left(b_{M-2}\right)} \frac{\bar{\phi}_{M}\left(a_{M}\right)}{\bar{\phi}_{M}\left(b_{M-1}\right)}=\frac{\bar{\phi}_{M-1}\left(a_{M-1}\right)}{\bar{\phi}_{M-1}\left(b_{M-2}\right)}<1,
$$


we get

$$
\left(\nu_{M-2}^{k+2}\left(X, b_{M-2}, t\right)\right)^{2} \varphi(t) \leq \frac{\bar{\phi}_{M-1}\left(a_{M-1}\right)}{\bar{\phi}_{M-1}\left(b_{M-2}\right)} \max \left\{E_{k}, E_{k+1}\right\} .
$$

Using the same techniques as the ones we use to achieve (3.12) and (3.13), we can prove that

$$
\left(\nu_{M-j}^{k+j}\left(X, b_{M-j}, t\right)\right)^{2} \varphi(t) \leq \frac{\bar{\phi}_{M-j+1}\left(a_{M-j+1}\right)}{\bar{\phi}_{M-j+1}\left(b_{M-j}\right)} \max \left\{E_{k}, \ldots, E_{k+j-1}\right\},
$$

where $\bar{\phi}_{M-j+1}$ is a strictly positive function satisfying

$$
\frac{\bar{\phi}_{M-j+1}\left(a_{M-j+1}\right)}{\bar{\phi}_{M-j+1}\left(b_{M-j}\right)}<1,
$$

with $j \in\{1, \ldots, M-1\}$.

Now, with (3.10), we can choose a strictly positive function $\bar{\phi}_{*}$ such that $\bar{\phi}_{*}\left(b_{M}\right)>\bar{\phi}_{*}\left(a_{M}\right)$. Then

$$
\left(\nu_{M}^{k}\left(X, b_{M}, t\right)\right)^{2} \bar{\phi}_{*}\left(b_{M}\right) \varphi(t) \leq \max _{\bar{D} \times[0, T]}\left\{\left(\nu_{M}^{k}\left(X, a_{M}, t\right)\right)^{2} \bar{\phi}_{*}\left(a_{M}\right) \varphi(t)\right\},
$$

and as a result,

$$
\left(\nu_{M}^{k}\left(X, b_{M}, t\right)\right)^{2} \bar{\phi}_{*}\left(b_{M}\right) \varphi(t) \leq \max _{\bar{D} \times[0, T]}\left\{\left(\nu_{M-1}^{k-1}\left(X, a_{M}, t\right)\right)^{2} \bar{\phi}_{*}\left(a_{M}\right) \varphi(t)\right\},
$$

which implies that

$$
\left(\nu_{M}^{k}\left(X, b_{M}, t\right)\right)^{2} \varphi(t) \leq \frac{\bar{\phi}_{*}\left(a_{M}\right)}{\bar{\phi}_{*}\left(b_{M}\right)} E_{k-1} .
$$

Step 2.2. Estimate of the left boundaries of the subdomains.

Consider the first domain. At the $k$-th step, 3.11 implies

$$
\left(\nu_{1}^{k}\left(X, x_{n}, t\right)\right)^{2} \tilde{\phi}_{1}\left(x_{n}\right) \varphi(t) \leq \max _{\bar{D} \times[0, T]}\left\{\left(\nu_{1}^{k}\left(X, b_{1}, t\right)\right)^{2} \tilde{\phi}_{1}\left(b_{1}\right) \varphi(t)\right\},
$$

where $\tilde{\phi}_{1}$ is a strictly positive function that will be chosen later.

Replacing $x_{n}$ by $a_{2}$, we get

$$
\left(\nu_{1}^{k}\left(X, a_{2}, t\right)\right)^{2} \varphi(t) \leq \frac{\tilde{\phi}_{1}\left(b_{1}\right)}{\tilde{\phi}_{1}\left(a_{2}\right)} \max _{\bar{D} \times[0, T]}\left\{\left(\nu_{1}^{k}\left(X, b_{1}, t\right)\right)^{2} \varphi(t)\right\} .
$$

Since $\nu_{1}^{k}\left(X, a_{2}, t\right)=\nu_{2}^{k+1}\left(X, a_{2}, t\right)$, then

$$
\left(\nu_{2}^{k+1}\left(X, a_{2}, t\right)\right)^{2} \varphi(t) \leq \frac{\tilde{\phi}_{1}\left(b_{1}\right)}{\tilde{\phi}_{1}\left(a_{2}\right)} \max _{\bar{D} \times[0, T]}\left\{\left(\nu_{1}^{k}\left(X, b_{1}, t\right)\right)^{2} \varphi(t)\right\} .
$$

We choose $\tilde{\phi}_{1}$ such that

$$
\frac{\tilde{\phi}_{1}\left(b_{1}\right)}{\tilde{\phi}_{1}\left(a_{2}\right)}<1
$$

and deduce

$$
\left(\nu_{2}^{k+1}\left(X, a_{2}, t\right)\right)^{2} \varphi(t) \leq \frac{\tilde{\phi}_{1}\left(b_{1}\right)}{\tilde{\phi}_{1}\left(a_{2}\right)} E_{k} .
$$


Second, on the second domain, at the $(k+1)$-th step, (3.11) leads to

$$
\begin{aligned}
& \left(\nu_{2}^{k+1}\left(X, x_{n}, t\right)\right)^{2} \tilde{\phi}_{2}\left(x_{n}\right) \varphi(t) \\
& \leq \max \left\{\max _{\bar{D} \times[0, T]}\left\{\left(\nu_{2}^{k+1}\left(X, b_{2}, t\right)\right)^{2} \tilde{\phi}_{2}\left(b_{2}\right) \varphi(t)\right\},\right. \\
& \left.\max _{\bar{D} \times[0, T]}\left\{\left(\nu_{2}^{k+1}\left(X, a_{2}, t\right)\right)^{2} \tilde{\phi}_{2}\left(a_{2}\right) \varphi(t)\right\}\right\},
\end{aligned}
$$

where $\tilde{\phi}_{2}$ is a strictly positive function and will be chosen later. Since

$$
\nu_{2}^{k+1}\left(X, a_{3}, t\right)=\nu_{3}^{k+2}\left(X, a_{3}, t\right),
$$

then

$$
\begin{aligned}
& \left(\nu_{3}^{k+2}\left(X, a_{3}, t\right)\right)^{2} \tilde{\phi}_{2}\left(a_{3}\right) \varphi(t) \\
& \leq \max \left\{\max _{\bar{D} \times[0, T]}\left\{\left(\nu_{2}^{k+1}\left(X, b_{2}, t\right)\right)^{2} \tilde{\phi}_{2}\left(b_{2}\right) \varphi(t)\right\},\right. \\
& \left.\max _{\bar{D} \times[0, T]}\left[\left(\nu_{2}^{k+1}\left(X, a_{2}, t\right)\right)^{2} \tilde{\phi}_{2}\left(a_{2}\right) \varphi(t)\right]\right\} .
\end{aligned}
$$

Hence

$$
\begin{array}{r}
\left(\nu_{3}^{k+2}\left(X, a_{3}, t\right)\right)^{2} \varphi(t) \\
\leq \max \left\{\frac{\tilde{\phi}_{2}\left(b_{2}\right)}{\tilde{\phi}_{2}\left(a_{3}\right)} \max _{\bar{D} \times[0, T]}\left\{\left(\nu_{2}^{k+1}\left(X, b_{2}, t\right)\right)^{2} \varphi(t)\right\},\right. \\
\left.\frac{\tilde{\phi}_{2}\left(a_{2}\right)}{\tilde{\phi}_{2}\left(a_{3}\right)} \max _{\bar{D} \times[0, T]}\left[\left(\nu_{2}^{k+1}\left(X, a_{2}, t\right)\right)^{2} \varphi(t)\right]\right\} .
\end{array}
$$

Combining this with (3.16), we get

$$
\left(\nu_{3}^{k+2}\left(X, a_{3}, t\right)\right)^{2} \varphi(t) \leq \max \left\{\frac{\tilde{\phi}_{2}\left(b_{2}\right)}{\tilde{\phi}_{2}\left(a_{3}\right)} E_{k+1}, \frac{\tilde{\phi}_{2}\left(a_{2}\right)}{\tilde{\phi}_{2}\left(a_{3}\right)} \frac{\tilde{\phi}_{1}\left(b_{1}\right)}{\tilde{\phi}_{1}\left(a_{2}\right)} E_{k}\right\} .
$$

Choosing $\tilde{\phi}_{2}$ such that

$$
\frac{\tilde{\phi}_{2}\left(b_{2}\right)}{\tilde{\phi}_{2}\left(a_{3}\right)}=\frac{\tilde{\phi}_{2}\left(a_{2}\right)}{\tilde{\phi}_{2}\left(a_{3}\right)} \frac{\tilde{\phi}_{1}\left(b_{1}\right)}{\tilde{\phi}_{1}\left(a_{2}\right)}<1,
$$

we then obtain

$$
\left(\nu_{3}^{k+2}\left(X, a_{3}, t\right)\right)^{2} \varphi(t) \leq \frac{\tilde{\phi}_{2}\left(b_{2}\right)}{\tilde{\phi}_{2}\left(a_{3}\right)} \max \left\{E_{k}, E_{k+1}\right\} .
$$

Using the same techniques as the ones that we used to derive (3.16) and (3.17), we can prove that

$$
\left(\nu_{j}^{k+j-1}\left(X, a_{j}, t\right)\right)^{2} \varphi(t) \leq \frac{\tilde{\phi}_{j-1}\left(b_{j-1}\right)}{\tilde{\phi}_{j-1}\left(a_{j}\right)} \max \left\{E_{k}, \ldots, E_{k+j-2}\right\},
$$

where $\tilde{\phi}_{j-1}$ is a strictly positive function satisfying

$$
\frac{\tilde{\phi}_{j-1}\left(b_{j-1}\right)}{\tilde{\phi}_{j-1}\left(a_{j}\right)}<1
$$

with $j \in\{1, \ldots, M-1\}$. 
Now, with (3.11), we can choose a strictly positive function $\tilde{\phi}_{*}$ such that $\tilde{\phi}_{*}\left(b_{1}\right)<$ $\tilde{\phi}_{*}\left(a_{1}\right)$ and get

$$
\left(\nu_{1}^{k}\left(X, a_{1}, t\right)\right)^{2} \tilde{\phi}_{*}\left(a_{1}\right) \varphi(t) \leq \max _{\bar{D} \times[0, T]}\left\{\left(\nu_{1}^{k}\left(X, b_{1}, t\right)\right)^{2} \tilde{\phi}_{*}\left(b_{1}\right) \varphi(t)\right\},
$$

which is equivalent to

$$
\left(\nu_{1}^{k}\left(X, a_{1}, t\right)\right)^{2} \tilde{\phi}_{*}\left(a_{1}\right) \varphi(t) \leq \max _{\bar{D} \times[0, T]}\left\{\left(\nu_{2}^{k-1}\left(X, b_{1}, t\right)\right)^{2} \tilde{\phi}_{*}\left(b_{1}\right) \varphi(t)\right\} .
$$

This implies

$$
\left(\nu_{M}^{k}\left(X, a_{1}, t\right)\right)^{2} \varphi(t) \leq \frac{\tilde{\phi}_{*}\left(b_{1}\right)}{\tilde{\phi}_{*}\left(a_{1}\right)} E_{k-1} .
$$

Step 2.3. Convergence result.

From (3.14), (3.15), (3.18) and (3.19), there exists $\gamma$ in $(0,1)$ such that

$$
\left(\nu_{l}^{k+M}\left(X, a_{l}, t\right)\right)^{2} \varphi(t) \leq \gamma \max \left\{E_{k}, \ldots, E_{k+M-1}\right\} \text {, for } l \in\{1, \ldots, M\},
$$

and

$$
\left(\nu_{l}^{k+M}\left(X, b_{l}, t\right)\right)^{2} \varphi(t) \leq \gamma \max \left\{E_{k}, \ldots, E_{k+M-1}\right\}, \text { for } l \in\{1, \ldots, M\} .
$$

Using (3.9) for $\phi \equiv 1$, we have that

$$
\begin{aligned}
& \left(\nu_{l}^{k}\left(X, x_{n}, t\right)\right)^{2} \varphi(t) \\
& \quad \leq \max \left\{\max _{\bar{D} \times[0, T]}\left\{\left(\nu_{l}^{k}\left(X, a_{l}, t\right)\right)^{2} \varphi(t)\right\}, \max _{\bar{D} \times[0, T]}\left\{\left(\nu_{l}^{k}\left(X, b_{l}, t\right)\right)^{2} \varphi(t)\right\}\right\} .
\end{aligned}
$$

Combining (3.20), (3.21) and (3.22), we get

$$
E_{k+M} \leq \gamma \max \left\{E_{k}, \ldots, E_{k+M-1}\right\} .
$$

Hence, $E_{k}$ tends to 0 as $k$ tends to infinity.

Step 3. Proof of convergence: for $l$ in $\{1, \ldots, M\}$, the sequence $\left\{e_{l}^{k}\right\}$ converges pointwisely to 0 as $k$ tends to infinity.

Since for $l$ in $\{1, \ldots, M\}$,

$$
\lim _{k \rightarrow \infty}\left\|\left(\nu_{l}^{k}\right)^{2} \varphi(t)\right\|_{C\left(\overline{\Omega_{l} \times(0, T)}\right)}=0
$$

the sequence $\left\{\left(\frac{\partial \epsilon_{l}^{k}}{\partial x_{n}}\right)^{2} \varphi(t)\right\}$ converges to 0 pointwisely and the sequence is bounded by a constant $M_{0}$. Since $\varphi$ is strictly positive on $[0, T]$, there exist positive constants $M_{1}, M_{2}$ such that $M_{1}<\varphi<M_{2}$. This means that the sequence $\left\{\left|\frac{\partial \epsilon_{l}^{k}}{\partial x_{n}}\right|\right\}$ converges to 0 pointwisely and is bounded by a constant $M_{3}$.

For $l=1$, with a fixed value of $(X, t)$, we get from the Lebesgue Dominated Convergence Theorem that for $x_{n}$ in $\left[a_{1}, b_{1}\right], \int_{a_{1}}^{x_{n}} \frac{\partial \epsilon_{1}^{k}}{\partial x_{n}}(X, \zeta, t) d \zeta$ converges to 0 as $k$ tends to infinity. Hence the sequence $\left\{\epsilon_{1}^{k}\left(X, x_{n}, t\right)-\epsilon_{1}^{k}\left(X, a_{1}, t\right)\right\}$ converges to 0 as $k$ tends to infinity. Since $\epsilon_{1}^{k}\left(X, a_{1}, t\right)=0$, the sequence $\left\{\epsilon_{1}^{k}\right\}$ converges to 0 pointwisely.

For $l=2$, with a fixed value of $(X, t)$, again by the Lebesgue Dominated Convergence Theorem, for $x_{n}$ in $\left[a_{2}, b_{2}\right]$, the sequence

$$
\left\{\int_{a_{2}}^{x_{n} t} \frac{\partial \epsilon_{2}^{k}}{\partial x_{n}}(X, \zeta, t) d \zeta\right\}
$$


converges to 0 as $k$ tends to infinity. Hence the sequence

$$
\left\{\epsilon_{2}^{k}\left(X, x_{n}, t\right)-\epsilon_{2}^{k}\left(X, a_{2}, t\right)\right\}
$$

converges to 0 as $k$ tends to infinity. Since

$$
\frac{\partial e_{2}^{k}}{\partial x_{n}}\left(X, a_{2}, t\right)+p e_{2}^{k}\left(X, a_{2}, t\right)=\frac{\partial e_{1}^{k-1}}{\partial x_{n}}\left(X, a_{2}, t\right)+p e_{1}^{k-1}\left(X, a_{2}, t\right),
$$

and the sequences $\left\{e_{1}^{k}\right\},\left\{\left|\frac{\partial \epsilon_{l}^{k}}{\partial x_{n}}\right|\right\}$ converge to 0 pointwisely for $l$ in $\{1, \ldots, M\}$, we can deduce that $\epsilon_{2}^{k}\left(X, x_{n}, t\right)$ converges to 0 as $k$ tends to infinity.

By similar processes, we can prove that for $l$ in $\{1, \ldots, M\}$, the sequence $\left\{e_{l}^{k}\right\}$ converges pointwisely to 0 as $k$ tends to infinity. This concludes the proof.

\section{Numerical Results}

We give here some numerical results to illustrate the convergence of the method. Here we use the Python module Optimism developed by Loic Gouarin [7]. This code uses the MPI library to solve domain decomposition problems and can handle any number of subdomains. The problem in our example is the following:

$$
\frac{\partial u}{\partial t}-\triangle u+\nabla \cdot u+u=0, \text { in }(0, T) \times \Omega,
$$

where $\Omega=(0,1) \times(0,1)$. The initial and boundary data are 0 .

The code uses the finite element method to solve the problem and a triangular mesh is used. The discretization steps in space and time are $d x=d y=d t=0.01$. We look only at the first iteration in time such that $T=d t$.

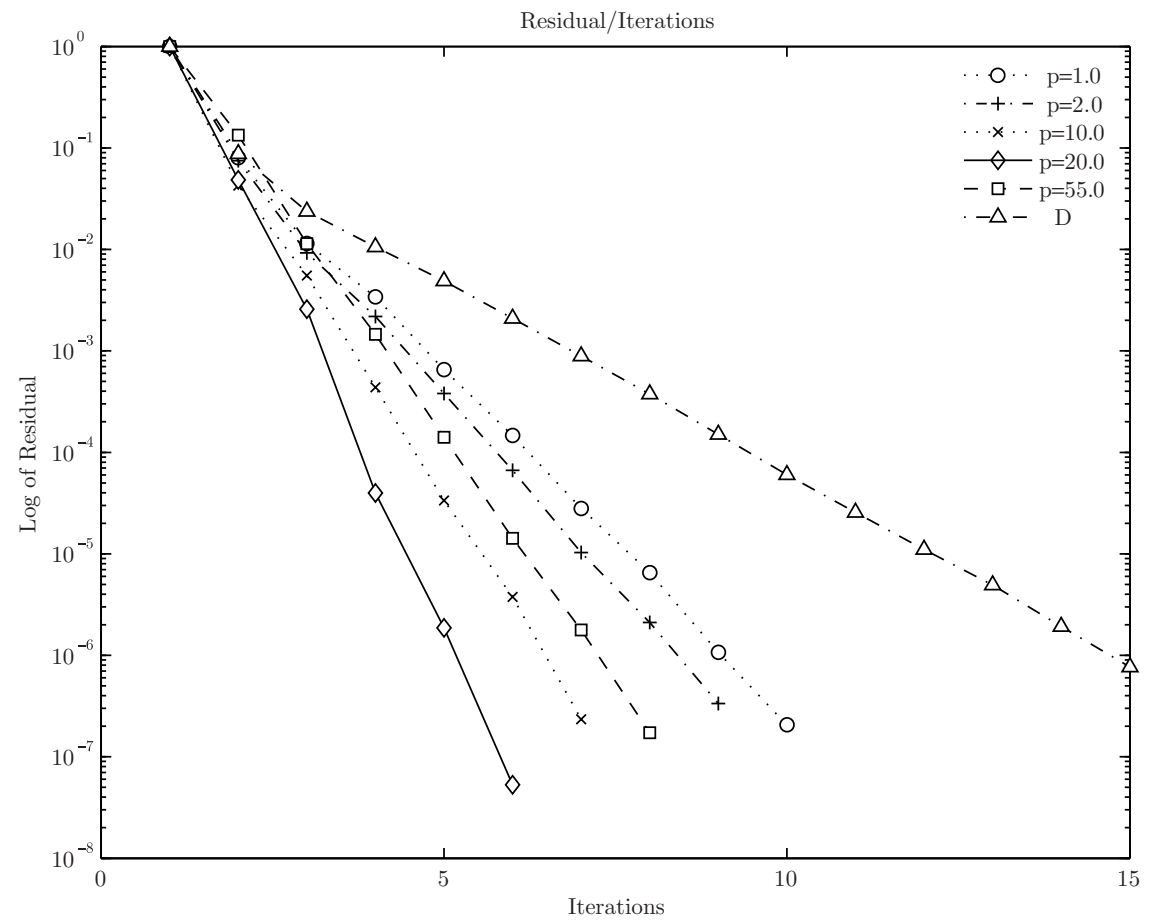


In our example, there are four subdomains $(M=4)$ and the decomposition into subdomains follows the $x$-direction. The overlapping length is $2 d x$. This means that the first subdomain is $[0,0.26] \times[0,1]$, the second one is $[0.24,0.51] \times[0,1]$, the third one is $[0.49,0.76] \times[0,1]$, and the fourth one is $[0.74,1] \times[0,1]$. We use random initial data $h^{0}$ on the boundaries $a_{l}$ and $b_{l}$.

We consider the performance of the algorithm for several values of $p$ including small and large ones: $1,2,10,20,55$. On the same graph, we also plot the performance of the algorithm with Dirichlet transmission condition. According to this test, the algorithm with Robin transmission conditions reaches the errors of $10^{-6}$ after at most 9 iterations, while the one with Dirichlet transmission conditions needs 15 iterations to reach this error.

\section{ACKNOWLEDGEMENTS}

The author would like to thank Professor Laurence Halpern for her kindness and support. The author would also like to express his gratitude to Loic Gouarin for his code and Gilles Scarella for his great help. The author is also grateful to the editor and the referee for carefully reading the paper and for useful remarks.

\section{RefEREnCES}

1. Jean-David Benamou and Bruno Desprès. A domain decomposition method for the Helmholtz equation and related optimal control problems. J. Comput. Phys., 136(1):68-82, 1997. MR.1468624(98c:65162)

2. D. Bennequin, M. J. Gander, and L. Halpern. A homographic best approximation problem with application to optimized Schwarz waveform relaxation. Math. Comp., 78(265):185-223, 2009. MR2448703 (2010g:65129)

3. Avner Friedman. Partial differential equations of parabolic type. Prentice-Hall Inc., Englewood Cliffs, N.J., 1964. MR0181836 (31:6062)

4. M. J. Gander and L. Halpern. Optimized Schwarz waveform relaxation methods for advection reaction diffusion problems. SIAM J. Numer. Anal., 45(2):666-697 (electronic), 2007. MR2300292(2008e:65291)

5. M. J. Gander, L. Halpern, and F. Nataf. Optimal convergence for overlapping and nonoverlapping Schwarz waveform relaxation. In Eleventh International Conference on Domain Decomposition Methods (London, 1998), pages 27-36 (electronic). DDM.org, Augsburg, 1999. MR 1827406

6. Martin J. Gander, Laurence Halpern, and Frederic Nataf. Optimized Schwarz methods. In Domain decomposition methods in sciences and engineering (Chiba, 1999), pages 15-27 (electronic). DDM.org, Augsburg, 2001. MR.1827519

7. Loic Gouarin, Python module Optimism. Personal communication.

8. L. Halpern, C. Japhet, and J. Szeftel. Discontinuous Galerkin and nonconforming in time optimized Schwarz waveform relaxation. In Proceedings of the Eighteenth International Conference on Domain Decomposition Methods, 2009. Domain Decomposition Methods in Science and Engineering, Vol. 19, pp. 133-140. Lect. Notes in Comput. Sci. Eng., Vol. 78, Springer, Heidelberg, 2011. MR2867652

9. Laurence Halpern and Jérémie Szeftel. Nonlinear nonoverlapping Schwarz waveform relaxation for semilinear wave propagation. Math. Comp., 78(266):865-889, 2009. MR2476563 (2010b:65202)

10. C. Japhet and Frédéric Nataf. The best interface conditions for domain decomposition methods: absorbing boundary conditions. In Absorbing boundaries and layers, domain decomposition methods, pages 348-373. Nova Sci. Publ., Huntington, NY, 2001. MR2039948

11. P.-L. Lions. On the Schwarz alternating method. I. In First International Symposium on Domain Decomposition Methods for Partial Differential Equations (Paris, 1987), pages 142. SIAM, Philadelphia, PA, 1988. MR972510(90a:65248) 
12. P.-L. Lions. On the Schwarz alternating method. II. Stochastic interpretation and order properties. In Domain decomposition methods (Los Angeles, CA, 1988), pages 47-70. SIAM, Philadelphia, PA, 1989. MR992003 (90e:65140)

13. P.-L. Lions. On the Schwarz alternating method. III. A variant for nonoverlapping subdomains. In Third International Symposium on Domain Decomposition Methods for Partial Differential Equations (Houston, TX, 1989), pages 202-223. SIAM, Philadelphia, PA, 1990. MR.1064345 (91g:65226)

14. Minh-Binh Tran. Convergence Properties of Overlapping Schwarz Domain Decomposition Algorithms. Submitted.

Basque Center for Applied Mathematics (BCAM), Alameda de Mazarredo, 14, E-48009 Bilbao, Basque Country, Spain

E-mail address: tbinh@bcamath.org 